\title{
Bubbles, Crashes and Efficiency with Double Auction Mechanisms ${ }^{\star}$
}

\author{
Jinpeng $\mathrm{Ma}^{1}$ and Qiongling $\mathrm{Li}^{2}$ \\ 1 Department of Economics, Rutgers University, Camden, NJ 08102 \\ jinpeng@crab.rutgers.edu \\ 2 Department of Mathematics, Rice University, 6100 Main St, Houston, TX 77005 \\ q140rice.edu
}

\begin{abstract}
"[S]ince equilibrium is a relationship between actions, and since the actions of one person must necessarily take place successively in time, it is obvious that the passage of time is essential to give the concept of equilibrium any meaning. This deserves mention since many economists appear to have been unable to find a place for time in equilibrium analysis and consequently have suggested that equilibrium must be conceived as timeless." $\quad$ F.A. Von Hayek (1937)
\end{abstract}

\begin{abstract}
We provide a quantitative boundary on the stepsizes of bid and ask of a double auction (DA) mechanism to answer two questions, when the DA mechanism is efficient and when it creates bubbles and crashes. The main result is that the ratio of the two stepsizes and their spread are the key factors for the DA mechanism to be efficient. Sentiment that leads to a swing in the spread and the ratio of the two stepsizes can result in prices to deviate from the intrinsic value equilibrium. These results are derived from a theoretical analysis of the DA mechanism built on the incremental subgradient method in Nedić and Bertsekas (2001).
\end{abstract}

Keywords: Double auction mechanism, incremental subgradient method, efficient markets hypothesis, investors' sentiment, job matching market, multiple objects.

${ }^{\star}$ We thank Mark Satterthwaite, an expert on DA mechanisms, for helpful communications. 